\title{
Dirty rotating black holes: regularity conditions on stationary horizons
}

\author{
I. V. Tanatarov ${ }^{1,2, *}$ and O. B. Zaslavskii ${ }^{2, \dagger}$ \\ ${ }^{1}$ Kharkov Intitute of Physics and Technology, \\ 1 Akademicheskaya, Kharkov 61108, Ukraine \\ ${ }^{2}$ Department of Physics and Technology, \\ Kharkov V.N. Karazin National University, \\ 4 Svoboda Square, Kharkov 61022, Ukraine
}

\begin{abstract}
We consider generic, or "dirty" (surrounded by matter), stationary rotating black holes with axial symmetry. The restrictions are found on the asymptotic form of metric in the vicinity of non-extremal, extremal and ultra-extremal horizons, imposed by the conditions of regularity of increasing strength: boundedness on the horizon of the Ricci scalar, of scalar quadratic curvature invariants, and of the components of the curvature tensor in the tetrad attached to a falling observer.
\end{abstract}

We show, in particular, that boundedness of the Ricci scalar implies the "rigidity" of the horizon's rotation in all cases, while the finiteness of quadratic invariants leads to the constancy of the surface gravity. We discuss the role of quasiglobal coordinate $r$ that is emphasized by the conditions of regularity. Further restrictions on the metric are formulated in terms of subsequent coefficients of expansion of metric functions by $r$. The boundedness of the tetrad components of curvature tensor for an observer crossing the horizon is shown to lead in the horizon limit to diagonalization of Einstein tensor in the frame of zero angular momentum observer on a circular orbit (ZAMO frame) for horizons of all degrees of extremality.

PACS numbers: 04.70.Bw, 04.20.-q, 04.70.Dy

*Electronic address: igor.tanatarov@gmail.com

${ }^{\dagger}$ Electronic address: zaslav@ukr.net 


\section{Contents}

I. Introduction

II. Basic formulas $\quad 5$

A. Types of horizons $\quad 5$

B. Axially symmetric space-times 6

III. Curvature invariants 6

A. Ricci scalar 6

B. Non-extremal horizons 8

1. Ricci scalar 8

2. Quadratic invariants 9

C. (Ultra)extremal horizons 9

1. Extremal case 9

2. An attempt to depart from analyticity 10

3. Quasiglobal coordinate 11

4. Ultraextremal case 12

D. Intermediate results: eliminating scalar curvature singularity 13

IV. Tetrad components of curvature tensor 13

A. Orbital ZAMO frame $\quad 14$

B. Falling ZAMO frame $\quad 16$

1. Choice of tetrad for a falling observer 16

2. Non-extremal horizons 17

3. Extremal horizons 18

4. Ultra-extremal horizons 19

5. Simplest case: $p=3 \quad 21$

V. Example: Kerr-Newman metric 22

A. Non-extremal horizon, $M^{2}>a^{2}+Q^{2} \quad 23$

B. Extremal horizon, $M^{2}=a^{2}+Q^{2}$

VI. On-horizon structure of the Einstein tensor 24

A. Direct calculation 24

B. Kinematic origin of the on-horizon structure of the Einstein tensor 26

$\begin{array}{ll}\text { VII. Summary and conclusion } & 27\end{array}$

$\begin{array}{ll}\text { Acknowledgments } & 29\end{array}$

VIII. Appendix: Einstein tensor in ZAMO frame 29

$\begin{array}{ll}\text { References } & 30\end{array}$ 


\section{INTRODUCTION}

The properties of the near-horizon geometry play an essential role in many important issues in gravitational physics. One of them is the constancy of the surface gravity $\kappa$ that is crucial for the validity of the laws of black hole mechanics [1] which were later recognized as laws of thermodynamics with $\kappa$ related to the temperature of the system [2]. Another property is the constancy of the angular velocity of a black hole $\omega_{H}$ that coincides with the horizon value of the metric function $\omega$ responsible for rotation (see details below) and allows one to consider a horizon as a solid rigidly rotating object. The fundamental properties of the near-horizon geometry established in [1] claim the constancy of $\kappa$ and $\omega_{H}$ but tell nothing of the asymptotic form of the metric and the rate with which $\omega-\omega_{H}$ approaches zero. Meanwhile, these details become very important, for example, in the relation between the symmetries of the near-horizon geometry and the universality of the Bekenstein-Hawking entropy. This served as a motivation for closer examination of the properties of the metric near the horizon for the "dirty" (surrounded by matter) black holes, undertaken in [3] for static and in [4] for stationary geometries. The aforementioned papers were mainly devoted to the nonextremal case. The ultraextremal one was only touched upon partially, while the extremal case escaped consideration at all. Roughly speaking, the extremal case corresponds to the degenerate horizons of the second order while the ultraextremal case implies the multiple horizon of the order three or higher. More explicit definition of such horizons in terms of the metric functions will be given in the text below.

In the present paper, we extend the results of [4] to the extremal and ultraextremal cases for which $\kappa=0$. We find the asymptotic expansion of the metric near the horizon compatible with the regularity of the geometry. It turns out that one cannot simply put $\kappa=0$ in the formulas derived in [4] since the asymptotic expansion for $\omega$ changes qualitatively. It is worth stressing that knowledge of such properties is necessary in a number of different physical problems. First of all, it concerns the issue of the black hole entropy [3], [4] which in the (ultra)extremal case becomes much more subtle and even contradictory [5]. The attempts to give a self-consistent description of the entropy in the extremal case were made for the spherically symmetric case [6], [7] but to generalize them to rotating geometries, detailed information on the asymptotic behavior of the metric near the horizon is needed.

Apart from this, it is required by a number of concrete physical and astrophysical applications. Let us give a couple of examples. Quite recently, the effect of acceleration of particles by rotating black holes (the BSW effect) was discovered for the Kerr extremal metric [8]. Later on, it was generalized to generic rotating dirty black holes, both for the extremal and nonextremal cases [9]. One of the key features of this work consisted in a proper account for the difference 
in the asymptotic behavior of the metric function $\omega$ responsible for rotation for extremal and nonextremal black holes (see eqs. (12) and (13) of [9]). The validity of such an expansion in the extremal case can be checked for the Kerr metric directly but it is important to have it in the general case as well. Another example is studying the limiting transition from the rotating so-called quasiblack hole - an object on the threshold of forming a horizon in a horizonless configuration - to the black hole limit [10]. Such a limit turns out to be well-defined only due to the validity of the same asymptotic expansion of $\omega$ (see eq.(5) in [11]). Apart from this, the properties of different kinds of observers in the vicinity of a black hole (orbiting around it or falling through the horizon) are important in the membrane formalism [12].

We find the restrictions which come from the two types of requirements. The first one is the finiteness of some curvature invariants imposed on the properties of the near-horizon geometry. This is done by examining the same invariants that were considered in [3], [4] for the nonextremal case. Thus our approach is purely geometric and, similarly to [3], [4] and contrary to [1], does not use any energy conditions imposed on matter near the horizon.

Meanwhile, there is also another regularity requirement not considered in [3], [4]. It states that in the frame attached to an observer crossing the horizon all curvature tensor components should be finite. If we want the vicinity of the horizon to be regular, the requirement under discussion imposes strong restrictions on the metric functions. If this requirement is violated, while the first one from the previous paragraph is satisfied, the corresponding space-time represents a so-called truly naked black hole (TNBH). Such objects, introduced and discussed in [13], [14] and [15], generalized some previous observations made in [16], [17]. From the mathematical viewpoint, their horizons are examples of the so-called nonscalar curvature singularities [18] (Chap. 8), [19].

The paper is organized as follows. In Sec. II, we give basic definitions of different types of horizons (nonextremal, extremal, ultraextremal) and describe briefly the metrics we consider. In Sec. III, we examine the Ricci scalar and the invariants quadratic with respect to the Ricci tensor and derive the restrictions on the metric functions that are necessary and sufficient for their finiteness. Also, we discuss the role of the requirement of the metric's analyticity near the horizon and that of the quasiglobal coordinate. In Sec. IV, the properties of the components of the Riemann tensor in the vicinity of the horizon are discussed for observers with zero angular momentum on circular orbits (OZAMOs) and for observers crossing the horizon. In sec. V we illustrate the general results on the example of Kerr-Newman vacuum solution. The on-horizon structure of the Einstein tensor in the OZAMO frame is considered in Sec. VI. Summary and Conclusion are given in Sec. VII. In Appendix (Sec. VIII) we give some useful formulas for the Einstein tensor in the OZAMO frame. 


\section{BASIC FORMULAS}

\section{A. Types of horizons}

In the simplest, spherically symmetric case of a black hole metric in terms of the so-called quasiglobal coordinate $r$

$$
d s^{2}=-f(r) d t^{2}+\frac{d r^{2}}{f(r)}+R^{2}(r) d \Omega^{2},
$$

the event horizon is the surface $r=r_{h}=$ const, on which $f(r) \rightarrow 0$. We assume that near the horizon $f \sim\left(r-r_{h}\right)^{p}$. For the horizon to be non-singular and allow analytic continuation into the inner region, $p$ must be integer (for more on this see [15]). The horizon is referred to as non-extremal if $p=1$ (so $r=r_{h}$ is a simple zero of $f(r)$ ), extremal if $p=2$, and ultraextremal if $p \geq 3$.

Making a change of variables from $r$ to the proper distance to the horizon $n$ and using the Gaussian normal coordinates we can write the metric in the neighborhood of the horizon as

$$
d s^{2}=-N^{2}(n) d t^{2}+d n^{2}+r^{2}(n) d \Omega^{2},
$$

where $N(n)$ is the lapse function. In the non-extremal case

$$
N(n) \sim \kappa n
$$

at the horizon, where $\kappa$ is the surface gravity. In the extremal case $n \sim \ln \left(r-r_{h}\right)$ and

$$
N \sim e^{-a n}
$$

( $a>0$ is a constant), and in the ultraextremal one

$$
N \sim n^{-k}
$$

with $k=\frac{p}{p-2}>0$. The condition $N=0$ on the horizon means that in the extremal and ultraextremal cases it is situated at infinite distance $n \rightarrow+\infty$.

In the absence of specific symmetries, the form of metric (1) is no longer valid. Then, the asymptotic dependence of the lapse function on the proper distance to the horizon can be taken as definition of the horizon type: it is non-extremal if $N(n) \sim n$, extremal if (4) holds, and ultraextremal in case relation (5) holds with $k>0$. 


\section{B. Axially symmetric space-times}

Consider a generic axially symmetric rotating black hole space-time. Its metric in the vicinity of the horizon can be written in terms of Gaussian normal coordinates as

$$
d s^{2}=-N^{2} d t^{2}+g_{\phi \phi}(d \phi-\omega d t)^{2}+d n^{2}+g_{z z} d z^{2}
$$

where $n$ is the proper distance to the horizon, on which $N=0$. Due to the symmetries, the metric functions here do not depend on $t$ and $\phi$. Instead of $n$ and $z$ one can use coordinates $r$ and $\theta$, similar to the Boyer-Lindquist ones for the Kerr metric. Throughout the paper we assume that the fundamental constants $G=c=\hbar=1$.

In what follows, we use notations for the indices

$$
\begin{aligned}
\mu, \nu, \lambda, \ldots=0,1,2,3 & =t, \phi, n, z ; \\
i, j, k, l, \ldots=1,2,3 & =\phi, n, z ; \\
A, B, C, \ldots=0,1 & =t, \phi ; \\
a, b, c, \ldots=\quad 2,3 & =\quad n, z .
\end{aligned}
$$

Under the listed assumption of symmetry, the nonvanishing Christoffel symbols for the metric (6) read

$$
\begin{array}{lll}
\Gamma_{A, a B}=\frac{1}{2} \partial_{a} g_{A B}, & \Gamma_{a, A B}=-\frac{1}{2} \partial_{a} g_{A B}, & \Gamma_{a, b c}={ }^{(2)} \Gamma_{a, b c} \\
\Gamma_{a B}^{A}=\frac{1}{2} g^{A D} \partial_{a} g_{B D}, & \Gamma_{A B}^{a}=-\frac{1}{2} g^{a b} \partial_{b} g_{A B}, & \Gamma_{b c}^{a}={ }^{(2)} \Gamma_{b c}^{a}
\end{array}
$$

where ${ }^{(2)} \Gamma_{a, b c}$ and ${ }^{(2)} \Gamma_{b c}^{a}$ are the Christoffel symbols for the two-dimensional metric $g_{a b}$.

\section{CURVATURE INVARIANTS}

\section{A. Ricci scalar}

In what follows, we need the expression for the Ricci scalar $R$. Let us consider the foliation of the space-time by hypersurfaces $t=$ const with the unit normal vectors $u_{\mu}=N \delta_{\mu}^{0}$. Then one can use the general formula (see, e.g., eq. (3.43) in the textbook [20])

$$
R={ }^{(3)} R+\left(K^{i j} K_{i j}-K^{2}\right)-2 \chi_{; \mu}^{\mu}
$$


Here semicolon denotes covariant derivative with respect to the metric $g_{\mu \nu} ;{ }^{(3)} R$ is the Ricci scalar of the slice $t=$ const, $K_{i j}$ is its extrinsic curvature tensor

$$
K_{i j}=u_{\mu ; \nu} e_{(i)}^{\mu} e_{(j)}^{\nu},
$$

where $e_{(i)}^{\mu}$ are the orthonormal basis vectors within the hypersurface, and

$$
\chi^{\nu} \equiv u^{\mu} u_{; \mu}^{\nu}-u^{\nu} u_{; \mu}^{\mu}
$$

It is convenient to choose $e_{(i)}^{\mu}$ along the coordinate axes $(\phi, n, z)$. Then, after straightforward calculations, we obtain that

$$
\begin{gathered}
\chi^{0}=\chi^{\phi}=0, \quad \chi^{a}=g^{a b} \partial_{b} \ln N \\
K_{\phi \phi}=K_{a b}=0, \quad K_{a \phi}=-\frac{g_{\phi \phi}}{2 N} \partial_{a} \omega .
\end{gathered}
$$

Rewriting $\chi_{; \mu}^{\mu}$ in terms of the three-metric $g_{i k}$, we can recast the scalar curvature in the form

$$
R={ }^{(3)} R+\frac{g_{\phi \phi}}{2 N^{2}}(\nabla \omega)^{2}-2 \frac{(3) \Delta N}{N}
$$

where ${ }^{(3)} \Delta N$ is the Laplacian calculated with respect to the metric $g_{i k}$ of the slice $t=$ const; $(\nabla \omega)^{2}=g^{a b}\left(\partial_{a} \omega\right)\left(\partial_{b} \omega\right)$ coincides for the metrics $g_{\mu \nu}, g_{i j}$ and $g_{a b}$, since $\omega$ does not depend on $t$ and $\phi$.

The third term comes from $\chi_{; \mu}^{\mu}$ and is the same as in the static case [3], while the second one appears due to non-zero extrinsic curvature of hypersurfaces $d t=0$ in axially symmetric space-times.

The right hand part of (18) in an explicit form is

$$
\begin{aligned}
R= & { }^{(3)} R+\frac{g_{\phi \phi}}{2 N^{2}}\left[\left(\partial_{n} \omega\right)^{2}+g_{z z}^{-1}\left(\partial_{z} \omega\right)^{2}\right]- \\
& -\left\{2\left(\frac{\partial_{n}^{2} N}{N}+g_{z z}^{-1} \frac{\partial_{z}^{2} N}{N}\right)+\partial_{n}\left(\gamma_{\phi}+\gamma_{z}\right) \cdot \frac{\partial_{n} N}{N}+g_{z z}^{-1} \partial_{z}\left(\gamma_{\phi}-\gamma_{z}\right) \cdot \frac{\partial_{z} N}{N}\right\},
\end{aligned}
$$

where $\gamma_{\phi}=\ln g_{\phi \phi}$ and $\gamma_{z}=\ln g_{z z}$; the terms in brackets come from extrinsic curvature, the ones in braces from $\chi_{\mu}^{; \mu}$. 


\section{B. Non-extremal horizons}

In this section we repeat the results obtained in [4] in order to lay down the scheme to be used later for (ultra)extremal horizons, write out the results explicitly for meaningful comparison and interpretation, and correct some minor errors made in the cited paper.

\section{Ricci scalar}

Let us consider $R$ in the vicinity of the horizon where, by definition, the lapse function has the asymptotic form $N \sim \kappa n(3)$. As all derivatives of $N$ are bounded, the term $\sim{ }^{(3)} \Delta N / N$ (the terms in braces in Eq. (19)) contains only divergences of the order $\sim 1 / n$. Meanwhile, assuming expansion of $\omega$ of the same type (we reserve notation $\omega_{i}$ to use it below)

$$
\omega(n, z)=\omega_{H}(z)+\hat{\omega}_{1}(z) n+\hat{\omega}_{2}(z) n^{2}+\ldots
$$

the terms originating from the contribution of external curvature contain divergences $\sim 1 / n^{2}$ :

$$
R=\frac{g_{\phi \phi}}{2}\left[\left(\frac{\partial_{n} \omega}{N}\right)^{2}+g_{z z}^{-1}\left(\frac{\partial_{z} \omega}{N}\right)^{2}\right]+O\left(\frac{1}{n}\right)
$$

As it is a sum of squares, in order for them not to diverge stronger than the remaining term $\sim 1 / n$, we need both $\partial_{n} \omega=O(\sqrt{n})$ and $\partial_{z} \omega=O(\sqrt{n})$, but taking into account that expansion (20) contains only integer powers of $n$, this is reduced to

$$
\partial_{n} \omega=O(N), \quad \partial_{z} \omega=O(N)
$$

when $N \rightarrow 0$. In terms of expansion coefficients, this means the rigidity of the horizon's rotation $\omega_{H}=$ const and also that $\omega_{1}=0$, so that expansion (20) reads

$$
\omega(n, z)=\omega_{H}+\hat{\omega}_{2}(z) n^{2}+\hat{\omega}_{3}(z) n^{3}+\ldots
$$

It can also be rewritten as a series by $N$

$$
\omega(n, z)=\omega_{H}+\tilde{\omega}_{2}(z) N^{2}+\tilde{\omega}_{3}(z) N^{3}+\ldots
$$

so $\partial_{z} \omega=O\left(N^{2}\right)$ also holds. Note that those are not sufficient conditions for $R$ to be finite, but further constraints are more conveniently obtained from the quadratic invariants. 


\section{Quadratic invariants}

Let us now consider the traceless part $Q_{\mu \nu}=R_{\mu \nu}-\frac{1}{4} R g_{\mu \nu}$ of the Ricci tensor squared

$$
R_{2} \equiv 4 Q_{\mu \nu} Q^{\mu \nu} \equiv 4 R_{\mu \nu} R^{\mu \nu}-R^{2}
$$

The expressions for the quadratic invariants do not seem to have an elegant form in terms of the three- and two-dimensional geometries, and in their explicit forms are rather lengthy, so we will not provide them here. In the limit $n \rightarrow 0$ for the non-extremal horizon, however, one can obtain

$$
R_{2}=\left[16\left(\frac{\partial_{n}^{2} N}{\kappa}\right)^{2}+\frac{8}{g_{z z}}\left(\frac{\partial_{z} N}{N}\right)^{2}+\left(\partial_{n} \gamma_{\phi}\right)^{2}+\left(\partial_{n} \gamma_{z}\right)^{2}\right]_{H} \cdot \frac{1}{n^{2}}+O\left(\frac{1}{n}\right)
$$

where subscript $H$ denotes that all the quantities are taken at the horizon. Note that the derivatives of $\gamma_{n, \phi}$ are by $n$, not by $z$ as given in [4]. This is again the sum of squares, and we obtain four conditions on the metric functions, which lead to their expansions of the form

$$
\begin{aligned}
N & =\kappa n+\hat{\kappa}_{3}(z) n^{3}+\ldots ; \\
g_{\phi \phi} & =g_{\phi H}(z)+\hat{g}_{\phi 2}(z) n^{2}+\ldots ; \\
g_{z z} & =g_{z H}(z)+\hat{g}_{z 2}(z) n^{2}+\ldots,
\end{aligned}
$$

where the surface gravity $\kappa$ is constant. Expansions (23) and (27)-(29) coincide with the corresponding results of [4] (eqs. (15)-(17) and (19)). It is straightforward to check that under those conditions the Ricci scalar (19) is regular. It turns out that the terms $\sim 1 / n$ in $R_{2}$ also vanish, so both $R_{2}$ and the Kretchman scalar $K r$ can be shown to be bounded.

\section{C. (Ultra)extremal horizons}

\section{Extremal case}

In the neighborhood of an extremal horizon the lapse function has the asymptotic form $N \sim e^{-a n}(4)$ and we assume expansion of all other metric functions in terms of $e^{-a n} \rightarrow 0$ (the horizon is at $n \rightarrow+\infty)$. Then the term $\sim{ }^{(3)} \Delta N / N$ in $R$ is regular, and the only potentially dangerous terms that can diverge at the horizon in this case are the ones in Eqs. (18) and (19) with derivatives of $\omega$, originating from the contribution of extrinsic curvature. Therefore we 
are again led to

$$
\partial_{n} \omega=O(N), \quad \partial_{z} \omega=O(N)
$$

for $N \rightarrow 0$, and the asymptotic expansion for $\omega$ reads

$$
\omega=\omega_{H}+\tilde{\omega}_{1}(z) N+O\left(N^{2}\right)
$$

where $\omega_{H}$ is constant. This is the same conclusion as for the nonextremal case (24), however, in contrast to nonextremal horizons, now there is no restriction on $\tilde{\omega}_{1}$.

Looking into the quadratic invariants, on substitution of (31) into (25) one can see that the invariant $R_{2}$ is finite on the horizon. We have checked that under the same assumptions the Kretschmann scalar is bounded as well, so the regularity of quadratic algebraic invariants of the curvature tensor provides no additional constraints.

\section{An attempt to depart from analyticity}

For the metric $(6), \kappa=\left(\frac{\partial N}{\partial n}\right)_{H}$. In what follows, we deal with the horizons for which $\kappa=0$. One can try to extend the definition of the extremal horizon and take, say, $a=a(z)$ in (4) instead of $a=$ const. This choice, however, is incompatible with the requirement of analyticity of the metric (1).

Analyticity can be defined in terms of the quasiglobal coordinate $r$ which behaves near the horizon as the Kruskal one [21], [22]. This is impossible for metric (6) in the whole spacetime, but nonetheless such a coordinate can be introduced approximately in the vicinity of the horizon: in the region where the Gaussian normal coordinates work, we can always pass from variable $n$ to $r=r(n)$, such that metric has the form

$$
d s^{2}=-N^{2}(r, z) d t^{2}+g_{\phi \phi}(d \phi-\omega d t)^{2}+\frac{d r^{2}}{A(r)}+g_{z z} d z^{2}
$$

with $A(r) \sim N^{2}(r, z) \sim r^{p}$ for $N \rightarrow 0$.

For nonextremal horizons $(p=1)$ this was done in Sec. VII of Ref. [23] but generalization to multiple horizons $(p \geq 2)$ is straightforward. The index $p$ should be integer if we want to have the metric analytical and extendable across the horizon. This rules out the dependence $a(z)$ in (4). Nonetheless, for completeness, we investigate below the case of $a(z)$ as well, relaxing the requirement of analyticity and relying on a weaker condition of the finiteness of curvature invariants only.

We assume the asymptotic form for the lapse function in the vicinity of the horizon $n \rightarrow+\infty$ 
is

$$
N=A(z) e^{-a(z) n}+B(z) e^{-2 a(z) n}+\ldots
$$

and examine whether such an asymptotic expression is compatible with the finiteness of $R$ and $R_{2}$.

When $a=a(z)$, the term ${ }^{(3)} \Delta N / N$ in $R(18)$ is not regular, but contains only polynomial divergences, and the exponential divergences can only be in the term with the derivatives of $\omega$. The conditions on the expansion of $\omega$, imposed in order to eliminate them, are reduced then to the ones obtained in the simpler case (31).

It turns out, that after substitution of (31) and (33) into $R_{2}$, all the exponential divergences in $R_{2}$ vanish, and only the polynomial ones remain. The worst possible remaining terms are proportional to $n^{4}$, as each power of $n$ arises from differentiation by $z$, and it can be shown that

$$
R_{2}=\frac{n^{4}}{4}\left[\frac{a^{\prime}(z)}{g_{\phi \phi} g_{z z}}\right]_{H}^{4} \cdot\left[\left(4-3 g_{\phi \phi} \omega_{1}^{2}\right)^{2}+2 g_{\phi \phi}^{2} \omega_{1}^{4}\right]_{H}+O\left(n^{3}\right) .
$$

The factor by $n^{4}$ is an explicitly positive quantity, so the divergence vanishes if and only if $a^{\prime}(z)=0$. Then all the lower degree divergences also vanish and $R_{2}$ is regular. The Kretschmann scalar in this case can be shown to be regular as well. Thus even the rather weak requirement of finiteness of $R_{2}$ forces us to reject the dependence of $a$ on $z$.

\section{Quasiglobal coordinate}

Given two radial-type coordinates, $n$ and $r$, it is natural to ask the question: in terms of which coordinate, $n$ or $r$ or some other, should we write expansions of the metric functions?

Note now that restrictions on the metric we have obtained till now, by ruling out scalar curvature singularities, are of two kinds: one is that some expansion coefficients are zero and the other is that some other expansion coefficients are constant on the horizon. The conditions of the first type appear in the non-extremal case only.

For the sake of simplicity, from now on we redefine the coordinate $r$ in such a way that $r_{h}=0$. For a non-extremal horizon $r \sim n^{2}$, so expansion by $n$, from which [4] and we started, is a generalization of expansion by $r$ : if $g_{\mu \nu}$ is expanded into a series by $r$, then $N(n)$ is a series with odd powers of $n$, while $\omega, g_{\phi \phi}$ and $g_{z z}$ are series with even powers of $n$. Thus the obtained regularity conditions of the first type actually tell us that the first terms of the 
assumed expansions by $n$ are actually only integer powers of $r$ :

$$
\begin{aligned}
& g_{\mu \nu}(r, z)=g_{\mu \nu}^{(h)}(z)+g_{\mu \nu}^{(1)} r+o(r) \\
& N^{2}(r, z)=\kappa^{2} r+\tilde{\kappa}_{2} r^{2}+o\left(r^{2}\right) .
\end{aligned}
$$

It is likely that if we considered scalar curvature invariants involving derivatives of higher order (e.g. $R_{\mu \nu \lambda \rho ; \sigma} R^{\mu \nu \lambda \rho ; \sigma}$ ), we would obtain further conditions demanding that terms with even powers of $n$ for $N$ and odd powers of $n$ for the other metric functions should be zero.

The idea that metric functions should be expanded in terms of the quasiglobal coordinate is also strengthened by the analysis of extremal horizon made in the previous paragraph. In this case expansions of $\omega$ or $g_{i i}$ in terms of powers of $n$ are just not viable, leading inevitably to scalar curvature singularity, while expansions in terms of $r$ are perfectly admissible.

In section IV we will obtain further indications that this reasoning is correct from consideration of boundedness of tetrad components of the curvature tensor in a tetrad attached to an observer crossing a non-extremal horizon.

\section{Ultraextremal case}

For the ultra-extremal case expansions in terms of $n$ and in terms of $r$ are in general incompatible, as opposed to non-extremal case, because $r \sim n^{m}$ with non-integer $m=-\frac{2}{p-2}$. In contrast to [4], we assume expansions of metric functions in terms of $r$ :

$$
\begin{aligned}
N^{2} & =\kappa_{p}(z) r^{p}+\kappa_{p+1}(z) r^{p+1}+\kappa_{p+2}(z) r^{p+2}+O\left(r^{p+2}\right) \\
\omega & =\omega_{H}(z)+\omega_{1}(z) r+\omega_{2}(z) r^{2}+O\left(r^{3}\right) \\
g_{\phi \phi} & =g_{\phi H}(z)+g_{\phi 1}(z) r+g_{\phi 2}(z) r^{2}+O\left(r^{3}\right) \\
g_{z z} & =g_{z H}(z)+g_{z 1}(z) r+g_{z 2}(z) r^{2}+O\left(r^{3}\right)
\end{aligned}
$$

Then the term $\sim{ }^{(3)} \Delta N / N$ in $R$ (18) is bounded, and in order to have the Ricci curvature bounded, the two conditions

$$
\partial_{n} \omega=O(N), \quad \partial_{z} \omega=O(N)
$$

must hold. The first one is satisfied automatically as soon as we assume expansions in terms of $r$ :

$$
\frac{\partial_{n} \omega}{N}=\frac{d r}{d n} \frac{\partial_{r} \omega}{N}=\frac{\sqrt{A}}{N} \partial_{r} \omega \sim \partial_{r} \omega=O(1) .
$$


The second one implies that the first $(q+1)$ expansion coefficients of $\omega$, starting from $\omega_{H}$ and ending with $\omega_{q}$ with $q=[(p+1) / 2]$ (brackets here denote integer part), do not depend on $z$, and depending on the parity of $p$ the corresponding term in $R$ is either $O(1)$ or $o(1)$ :

$$
\begin{array}{ll}
\omega=\omega_{H}+\ldots+\omega_{q-1} r^{q-1}+\omega_{q}(z) r^{q}+\ldots ; \\
p=2 q-1: \quad N \sim r^{q-1 / 2} \Rightarrow \partial_{z} \omega \sim r^{q}=o(N) ; \\
p=2 q \quad: \quad N \sim r^{q} \Rightarrow \partial_{z} \omega \sim r^{q}=O(N) .
\end{array}
$$

The general form of expansions for quadratic invariants is rather complicated, because the series starting with $r^{0}$ and ones starting with $r^{p}$ get intermixed. However, one can check that for specific cases $p=3, \ldots, 8$, assuming the restrictions on $\omega$ obtained above hold, both $R_{2}$ and $K$ are regular. Thus, just as in the extremal case, no additional constraints are obtained.

\section{Intermediate results: eliminating scalar curvature singularity}

As mentioned above, the restrictions on metric obtained from the demand of boundedness of scalar curvature invariants for any type of horizon are of two kinds. Those of the first kind, when some expansion coefficients are zero, can be summarized in the following way: expansion of the metric has the form $(35,36)$, i.e. the first several terms are a part of a series by the quasiglobal coordinate $r \sim n^{2}$ rather than by $n$. This is obtained for non-extremal horizons, while for (ultra-)extremal ones we assume expansion by $r$ from the very beginning, with $\kappa=0$ and the first term $\sim r^{p}$.

The restrictions of the second kind are reduced to 1) restrictions on $\omega(41)$

$$
\partial_{z} \omega=O(N)
$$

which enforce the "rigidity" of the horizon's rotation (in non-extremal case this also implies $\partial_{z} \omega=O\left(N^{2}\right)$ ), and 2) the constancy of the surface gravity (which holds by definition for (ultra)extremal horizons with $\kappa=0$ ).

\section{TETRAD COMPONENTS OF CURVATURE TENSOR}

In the preceding section, we considered the conditions imposed on the metric by the finiteness of two curvature invariants. Meanwhile, more detailed information about the properties of the metric near the horizon is required. One may ask, what is the behavior of the gravitational characteristics which can be directly measured by an observer? In other words, we are interested 
in the components of the curvature tensor in the tetrad frame attached to different observers.

In particular, we consider two classes of them. The observer of the first type is orbiting a black hole outside of the horizon on a circular orbit, with its angular momentum equal to zero. Such observers were introduced in [24] for the case of the Kerr metric and are usually referred to as ZAMOs (zero angular momentum observers), but we prefer to be more specific and will call them "orbital zero angular momentum observers", or OZAMOs for brevity. The observer of the second type falls through the horizon inside the black hole, freely or with finite proper acceleration, with conserving energy and angular momentum also equal to zero. We will call them "falling zero angular momentum observers", or FZAMOs for brevity.

Physically, the conditions on the metric which can be derived from the finiteness of the tetrad components of the curvature tensors can be different for OZAMOs and FZAMOs. OZAMO frame is the most natural generalization of the static frame in a static space-time to stationary metric. In the static case, the Kretschmann invariant $K r$ can be written in terms of the separate components of the curvature tensor as a sum of squares, so the finiteness of $K r$ requires the finiteness of each of them. Correspondingly, any algebraic invariant composed from the curvature tensor will be also finite. In the stationary case, the expression for $K r$ includes terms with different signs because of mixed components (with indices 0 and $\phi$ ), so the general picture is much more complicated.

Even in the static case, the components of the curvature tensor in the FZAMO frame responsible for tidal forces can be significantly enhanced near the horizon [16], [17]. This amounts to infinite values of some of them [13], [14] which remains compatible with the finiteness of curvature invariants. The corresponding objects represent the so-called truly naked black holes. If we want the metric to be completely regular, we should exclude such space-times. However, for this purpose, it is insufficient to examine the curvature invariants and the information about properties of FZAMO is required.

Thus we need to clarify the connection between (i) the finiteness of curvature invariants and the conditions obtained in the OZAMO frame, (ii) derive the corresponding conditions for FZAMO which are expected to give in general additional constraints on the properties of the metric.

\section{A. Orbital ZAMO frame}

First of all, let us consider the components of curvature tensor $R_{\mu \nu \rho \sigma}$ in the coordinate frame (t $\phi n z)$. Using the Gauss-Codazzi equations [20] for the $R_{i k l m}$ components (without zero 
indices), we get

$$
R_{i k l m}={ }^{(3)} R_{i k l m}+\left(K_{i l} K_{k m}-K_{i k} K_{l m}\right)
$$

thus using (16) we have explicitly

$$
\begin{aligned}
& R_{a b c d}={ }^{(3)} R_{a b c d} \\
& R_{a \phi b \phi}={ }^{(3)} R_{a \phi b \phi}-\frac{g_{\phi \phi}^{2}}{4} \frac{\partial_{a} \omega}{N} \frac{\partial_{b} \omega}{N}
\end{aligned}
$$

while $R_{\phi a b c}=0$ due to symmetry. Thus the conditions (22) are necessary for $R_{\mu \nu \rho \sigma}$ to be bounded, and direct calculation of the other components shows that they are also sufficient for that:

$$
R_{\mu \nu \rho \sigma}=O(1) \quad \Leftrightarrow \quad R=O(1)
$$

This fact does not seem to have any deep physical meaning by itself, though, as the finiteness of curvature tensor in the given (badly behaved) coordinate frame is irrelevant to regularity of geometry or observables.

Now let us consider an OZAMO - zero angular momentum observer on a circular orbit $n=$ const, $z=$ const. The corresponding frame is defined by the tetrad

$$
h_{(0)}=-N d t, \quad h_{(1)}=\sqrt{g_{\phi \phi}}(d \phi-\omega d t), \quad h_{(2)}=d n, \quad h_{(3)}=\sqrt{g_{z z}} d z .
$$

The tetrad components of the curvature tensor in this frame will be denoted by overall tildes, i.e. $\tilde{R}_{\mu \nu \rho \sigma}$. As the components in the coordinate frame $R_{\mu \nu \rho \sigma}$ are bounded and do not generally tend to zero, and there is a divergent factor in $h_{(0)}^{\mu} \sim 1 / N$, naive expectations would be that $\tilde{R}_{0 i 0 j} \sim h_{(0)}^{\mu} h_{(0)}^{\nu} R_{\mu i \nu j}$ diverge as $1 / N^{2}$ and $\tilde{R}_{0 i j k}$ as $1 / N$. However, as we show below, this is not the case.

The components $\tilde{R}_{a \phi b \phi}$ in the OZAMO frame differ from $R_{a \phi b \phi}(49)$ only by a factor of the order of unity, thus the conditions $(22)$ are at least necessary for the boundedness of $\tilde{R}_{\mu \nu \rho \sigma}$. Assuming they hold, direct calculation shows that all the other components of the curvature tensor in the OZAMO frame are finite with the possible exception of

$$
\begin{aligned}
& \tilde{R}_{0123}=-\frac{1}{2} \sqrt{\frac{g_{\phi \phi}}{g_{z z}}} \frac{\partial_{z} \omega}{N} \frac{\partial_{n} N}{N}+O(1) \\
& \tilde{R}_{0312}=+\frac{1}{2} \sqrt{\frac{g_{\phi \phi}}{g_{z z}}} \frac{\partial_{z} \omega}{N} \frac{\partial_{n} N}{N}+O(1)
\end{aligned}
$$

However, as $\partial_{n} N / N \sim \partial_{r} N \sim r^{p / 2-1}$, in the (ultra-)extremal case $p \geq 2$ those are also regular, so no additional constraints appear; and in the non-extremal case the two conditions (22) 
actually imply that $\partial_{z} \omega=O\left(N^{2}\right)$ (see (23)), so there are no additional constraints in this case either. Thus for all types of horizons

$$
\tilde{R}_{\mu \nu \rho \sigma}=O(1) \quad \Leftrightarrow \quad R=O(1)
$$

We see therefore that in the vicinity of a regular horizon an OZAMO observer always experiences finite tidal forces, even though his acceleration itself may diverge (at a non-extremal horizon).

\section{B. Falling ZAMO frame}

\section{Choice of tetrad for a falling observer}

If there's a Killing vector $\xi^{\mu}$, then the quantity $\xi^{\mu} u_{\mu}$ is conserved for a free-falling particle with 4-velocity $u^{\mu}$. Moreover, conservation of $u^{\mu} \xi_{\mu}$ along the worldline of any particle, not necessarily free falling, is equivalent to its acceleration along the Killing vector field being zero:

$$
a^{\nu} \xi_{\nu}=\left(u^{\mu} \nabla_{\mu} u^{\nu}\right) \xi_{\nu}=u^{\mu} \nabla_{\mu}\left(u^{\nu} \xi_{\nu}\right)-u^{\mu} u^{\nu} \nabla_{\mu} \xi_{\nu}=u^{\mu} \nabla_{\mu}\left(u^{\nu} \xi_{\nu}\right)
$$

As the two Killing vectors for axially symmetric metric are $\partial_{t}$ and $\partial_{\phi}$, the two corresponding quantities that conserve on geodesics are energy $E=-u_{t}$ and angular momentum $L=u_{\phi}$ (for particles of unit mass, which will be considered hereafter).

Let us consider FZAMO, i.e. the observer with zero angular momentum and conserving energy that is falling towards the horizon relative to an OZAMO. According to what is written above, the $t$ and $\phi$ components of his acceleration are zero. So, this class of observers obviously includes some of the free-falling ones, with corresponding values of $E$ and $L$.

We attach the tetrad to FZAMOs and investigate the restrictions on the metric that would ensure that the curvature tensor components in this tetrad are bounded. The tetrad can be built in three steps:

- First, we take the tetrad of OZAMO frame (51).

- Second, we rotate the frame in the $n-z$ plane by angle $\theta$

$$
\tilde{e}_{(2)}=h_{(2)} \cos \theta+h_{(3)} \sin \theta, \quad \tilde{e}_{(3)}=-h_{(2)} \sin \theta+h_{(3)} \cos \theta .
$$


- Finally, make a boost in the direction of $\tilde{e}_{(2)}$ :

$$
e_{(0)}=\gamma\left(h_{(0)}+v \tilde{e}_{(2)}\right), \quad e_{(1)}=h_{(1)}, \quad e_{(2)}=\gamma\left(\tilde{e}_{(2)}+v h_{(0)}\right), \quad e_{(3)}=\tilde{e}_{(3)},
$$

where

$$
\gamma=\frac{E}{N}
$$

is the Lorentz factor of the observer with respect to OZAMO frame, $v=\sqrt{1-1 / \gamma^{2}}$ is the physical velocity, $v_{n}=v \cos \theta$ and $v_{z}=v \sin \theta$ are its spatial components.

We do not consider below particles with $E=0$. Those are a specific case of critical particles, defined in general by relation $E-\omega_{H} L=0$, which are special and give rise to many interesting phenomena, such as the BSW effect [8].

We will also impose one additional restriction on the observers:

$$
\theta=O(N)
$$

It always holds in the spherically symmetric case due to additional constant of motion. In the axially symmetric non-extremal case it can be shown to follow explicitly from the boundedness of a particle's scalar acceleration. Extremal, and all the more so ultra-extremal general axially symmetric metrics in principle allow motion even with $\theta=O(1)$. However, such particles and corresponding metrics are also in a sense quite special, unique to extremal horizons, and should be investigated separately. We will not go into details here (they will be reported elsewhere), but instead, though it may seem rather arbitrary, just assume (59) in all cases.

\section{Non-extremal horizons}

The components of curvature tensor in the tetrad frame $\left\{e_{(i)}\right\}$ (57), attached to FZAMO, will be denoted by overall hats $\hat{R}_{\mu \nu \rho \sigma}$. Assuming expansions $(23,27,28,29)$, the components 
responsible for the tidal forces experienced by the observer are as follows near the horizon:

$$
\begin{aligned}
& \hat{R}_{0101}=-\frac{3 E^{2}}{2 \kappa^{2} g_{\phi H}} \frac{\hat{g}_{\phi 3}}{n}+O(1) ; \\
& \hat{R}_{0202}=O(1) ; \\
& \hat{R}_{0303}=-\frac{3 E^{2}}{2 \kappa^{2} g_{\phi H}} \frac{\hat{g}_{z 3}}{n}+O(1) ; \\
& \hat{R}_{0102}=-\frac{3 E}{2 \kappa^{2}} \sqrt{g_{\phi H}} \frac{\hat{\omega}_{3}}{n}+O(1) ; \\
& \hat{R}_{0103}=\frac{E^{2} \sqrt{g_{\phi H}}}{2 \kappa^{3} \sqrt{g_{z H}}}\left(\frac{\theta}{n} 3 \sqrt{g_{z H}} \hat{\omega}_{3}-\hat{\omega}_{3}^{\prime}\right) \frac{1}{n}+O(1) ; \\
& \hat{R}_{0203}=O(1) .
\end{aligned}
$$

The necessary and sufficient conditions for them to be bounded are

$$
\hat{g}_{z 3}=\hat{g}_{\phi 3}=\hat{\omega}_{3}=0
$$

It can be checked that all the other components of curvature tensor are also bounded under these assumptions.

Together with conditions $(35,36)$, already taken into account, these restrictions can be reformulated especially simply in terms of quasiglobal coordinate:

$$
g_{\mu \nu}=g_{\mu \nu}^{(h)}+g_{\mu \nu}^{(1)} r+g_{\mu \nu}^{(2)} r^{2}+o\left(r^{2}\right)
$$

Thus when we excluded scalar curvature singularity, we had to assume that metric is expanded in terms of $r$ instead of $n$ up to at least the terms $\sim r \sim n^{2}(35)$, with $N^{2}$ up to $\sim r^{2} \sim n^{4}(36)$, and now, excluding also truly naked black holes, we have to demand that the same condition holds for all components of $g_{\mu \nu}$ at least up to terms $\sim r^{2} \sim n^{4}$.

On the other hand, we see that there is no restriction on the first non-zero expansion coefficients of $g_{\mu \nu}$ after the leading terms - they are allowed to be functions of $\mathrm{z}$ - and in this sense the restrictions obtained from the OZAMO and FZAMO frames are the same. We will see below that in the (ultra)extremal case this is not so.

\section{Extremal horizons}

In order to extract the necessary and sufficient conditions of regularity of the curvature tensor in the FZAMO tetrad in the extremal and ultra-extremal cases it appears not to be enough to consider only the tidal forces $\hat{R}_{0 i 0 j}(i, j=1,2,3)$. So we look at all the components 
in the order that allows us to extract those conditions most efficiently.

Assuming the only restriction obtained for extremal horizons from the demand of curvature invariants' regularity $\omega_{H}=$ const (31), the smallness of $\theta$ (59) and expansion of metric in terms of $r$ of general form (37)-(40) for extremal horizons, with $p=2$, so that

$$
N^{2}=\kappa_{2}(z) r^{2}+\kappa_{3}(z) r^{3}+O\left(r^{4}\right)
$$

the asymptotics of some of the tetrad components are given by

$$
\begin{aligned}
\hat{R}_{0101} \sim \hat{R}_{1212} \sim \hat{R}_{0112} & \sim \frac{1}{r^{2}}\left[g_{\phi H}^{\prime} \kappa_{2}^{\prime}+g_{\phi H}^{2}\left(\omega_{1}^{\prime}\right)^{2}\right] ; \\
\hat{R}_{0103} \sim \hat{R}_{0123} \sim r \hat{R}_{0312} \sim r \hat{R}_{1223} & \sim \frac{1}{r^{2}}\left[2 \kappa_{2} \omega_{1}^{\prime}-\omega_{1} \kappa_{2}^{\prime}\right] ; \\
\hat{R}_{0203} \sim \hat{R}_{0223} & \sim \frac{1}{r}\left[2 \kappa_{2}^{\prime}-3 g_{\phi H} \omega_{1} \omega_{1}^{\prime}\right] \\
\hat{R}_{0113} & \sim \frac{\omega_{1} \omega_{1}^{\prime}}{r}
\end{aligned}
$$

For their boundedness it is necessary that $\omega_{1}^{\prime}=\kappa_{2}^{\prime}=0$. Under those conditions the expressions for $\hat{R}_{\mu \nu \rho \sigma}$ are further simplified and we see, in particular, that

$$
\hat{R}_{0101} \sim \hat{R}_{0103} \sim \hat{R}_{1212} \sim \hat{R}_{0112} \sim \frac{\kappa_{3}^{\prime}}{r}
$$

Thus the necessary conditions are

$$
\omega_{1}^{\prime}=\kappa_{2}^{\prime}=\kappa_{3}^{\prime}=0
$$

They can be rewritten as

$$
\partial_{z} \omega=O\left(N^{2}\right), \quad \frac{\partial_{z} N^{2}}{N^{2}}=O\left(N^{2}\right)
$$

This set now can be verified to be sufficient for all the tetrad components of the curvature tensor to be bounded. They are clearly more strict conditions on the metric than just those that are necessary for the boundedness of scalar invariants (30).

\section{Ultra-extremal horizons}

In this case, it is more convenient to work in terms of metric functions and their asymptotics, rather than in terms of their expansions, as the series starting with $r^{0}$ and $r^{p}$ are multiplied 
and divided, so the resulting series for arbitrary $p$ are hard to deal with. Thus we do the following. First, we calculate the exact expressions for the components of the curvature tensor in the FZAMO tetrad, and inspect them for divergences in assumptions (59) and

$$
N^{2} \sim A(r) \sim r^{p}, \quad p>2, \quad \partial_{z} \omega=O(N)
$$

where the last condition comes from the finiteness of curvature scalars according to (46). Then starting from some component we write out the explicit condition of its boundedness in the form $f\left(g_{\mu \nu}, \partial_{\lambda} g_{\mu \nu}\right)=O(1)$, where $f$ includes all the potentially divergent terms. This condition is then used to simplify the expressions for the other components, and allows us to write out the next condition that is needed for the finiteness of the next component. This procedure is repeated until all the components are finite, and this gives us, by construction, the set of necessary and sufficient conditions for $\hat{R}_{\mu \nu \rho \sigma}$ to be regular. The choice of specific succession can significantly simplify the conditions and their subsequent reduction. Our choice leads to the following sequence:

1. $\hat{R}_{0113}: \partial_{r} \omega \cdot \partial_{z} \omega=O\left(N^{2}\right)$;

2. $\quad \hat{R}_{0101}: \partial_{z} \gamma_{\phi} \frac{\partial_{z} N^{2}}{N^{2}}+g_{\phi \phi}\left(\frac{\partial_{z} \omega}{N}\right)^{2}=O\left(N^{2}\right)$

3. $\hat{R}_{0203}: \frac{\partial_{z} N^{2}}{N^{2}}=O(r) \Leftrightarrow \kappa_{p}^{\prime}=0 ;$

4. $\quad \hat{R}_{0303}: \partial_{z}\left(\gamma_{z}-3 \gamma_{\phi}\right) \frac{\partial_{z} N^{2}}{N^{2}}+\left(\frac{\partial_{z} N^{2}}{N^{2}}\right)^{2}-2 \frac{\partial_{z}^{2} N^{2}}{N^{2}}=O\left(N^{2}\right) ;$

5. $\hat{R}_{0313}: \partial_{z}\left(\gamma_{z}-3 \gamma_{\phi}\right) \cdot \partial_{z} \omega-2 \partial_{z}^{2} \omega+\frac{\partial_{z} N^{2}}{N^{2}} \partial_{z} \omega=O\left(N^{2}\right)$;

6. $\hat{R}_{0103}: \partial_{r}\left(\gamma_{z}-3 \gamma_{\phi}\right) \cdot \partial_{z} \omega-2 \partial_{r} \partial_{z} \omega+2 \frac{\partial_{r} N^{2}}{N^{2}} \partial_{z} \omega-\frac{\partial_{z} N^{2}}{N^{2}} \partial_{r} \omega=O\left(N^{2}\right)$.

Note that if (74) hold, then the six conditions (76-81) are also satisfied. So (74) are sufficient but not in general necessary conditions for the ultra-extremal case.

It should be noted that the order of the obtained restrictions on metric is not what would be expected from naive considerations: while the components of curvature tensor in OZAMO frame are bounded, which is ensured by conditions $\partial_{z} \omega=O(N)$ etc. (and which in itself is not expected from naive considerations), making the boost into the FZAMO frame with Lorentz factor $\sim 1 / N$, we introduce divergent factors, the worst of which are $\sim 1 / N^{2}$; so one would expect that regularity conditions change to e.g. $\partial_{z} \omega=O\left(N^{3}\right)$. The correct conditions turn out to be much softer, and the restriction (59) is essential here. 


\section{Simplest case: $p=3$}

Let us obtain the explicit restriction on the metric that the six conditions (76-81) imply in terms of the metric functions' expansion coefficients by $r$ (37-40) in the simplest case of ultra-extremal horizon, for $p=3$. Condition 2 is equivalent to $\kappa_{p}^{\prime}=0$; after substitution of (37-40) into the left hand side of the remaining five, we demand that all terms up to $\sim r^{2}$ are equal to zero, as in this case $O\left(N^{2}\right)$ is $O\left(r^{3}\right)$. After some algebra we obtain that there are three possible variants.

1. The first is the one to be expected, with $\partial_{z} N^{2}=O\left(N^{4}\right)$ and $\partial_{z} \omega=O\left(N^{2}\right)$, which in terms of expansion coefficients means

$$
\begin{aligned}
& \kappa_{3}^{\prime}=\kappa_{4}^{\prime}=\kappa_{5}^{\prime}=0 ; \\
& \omega_{H}^{\prime}=\omega_{1}^{\prime}=\omega_{2}^{\prime}=0
\end{aligned}
$$

with no other constraints. The expansions themselves are then

$$
\begin{gathered}
N^{2}(r, z)=\underline{\kappa_{3}} r^{3}+\underline{\kappa_{4}} r^{4}+\underline{\kappa_{5}} r^{5}+\kappa_{6}(z) r^{6}+\ldots ; \\
\omega(r, z)=\underline{\omega_{H}}+\underline{\omega_{1}} r+\underline{\omega_{2}} r^{2}+\omega_{3}(z) r^{3}+\ldots ;
\end{gathered}
$$

with $g_{\phi \phi}$ and $g_{z z}$ given by the general formulas (39), (40). Here and below in this section we underline the coefficients in the expansions that do not depend on $z$.

2. The second possible variant is more exotic:

$$
\begin{aligned}
& \kappa_{3}^{\prime}=\kappa_{4}^{\prime}=0, \quad \text { but } \quad \kappa_{5}^{\prime} \neq 0 ; \\
& \omega_{H}^{\prime}=\omega_{2}^{\prime}=0, \quad \omega_{1}=0 ; \\
& g_{\phi H}^{\prime}=0 ; \\
& g_{z H}=\text { const } \cdot\left(\kappa_{5}^{\prime}\right)^{2},
\end{aligned}
$$

so that the expansions (37-40) read

$$
\begin{aligned}
N^{2}(r, z) & =\underline{\kappa_{3}} r^{3}+\underline{\kappa_{4}} r^{4}+\kappa_{5}(z) r^{5}+\ldots ; \\
\omega(r, z) & =\underline{\omega_{H}}+\underline{\omega_{2}} r^{2}+\omega_{3}(z) r^{3}+\ldots ; \\
g_{\phi \phi}(r, z) & =\underline{g_{\phi H}}+g_{\phi 1}(z) r+\ldots ; \\
g_{z z}(r, z) & =\text { const } \cdot\left(\kappa_{5}^{\prime}\right)^{2}+g_{z 1}(z) r+\ldots
\end{aligned}
$$


3. Lastly, there is one more variant, most exotic, with expansion coefficients

$$
\begin{aligned}
& \kappa_{3}^{\prime}=0, \quad \text { but } \quad \kappa_{4}^{\prime} \neq 0 \\
& \omega_{H}^{\prime}=0, \quad \omega_{1}=\omega_{2}=0 \\
& g_{\phi H}^{\prime}=g_{\phi 1}^{\prime}=0, \\
& g_{z H}=C_{1} \cdot\left(\kappa_{4}^{\prime}\right)^{2}, \quad g_{z 1}=g_{z H}\left(2 \frac{\kappa_{5}^{\prime}}{\kappa_{4}^{\prime}}-\frac{\kappa_{4}}{\kappa_{3}}+C_{2}\right),
\end{aligned}
$$

where $C_{1,2}$ are constants, such that the metric functions are

$$
\begin{aligned}
N^{2}(r, z) & =\underline{\kappa_{3}} r^{3}+\kappa_{4}(z) r^{4}+\kappa_{5}(z) r^{5}+\ldots ; \\
\omega(r, z) & =\underline{\omega_{H}}+\omega_{3}(z) r^{3}+\omega_{4}(z) r^{4}+\ldots ; \\
g_{\phi \phi}(r, z) & =\underline{g_{\phi H}}+\underline{g_{\phi 1}} r+g_{\phi 2}(z) r^{2}+\ldots ; \\
g_{z z}(r, z) & =C_{1} \cdot\left(\kappa_{4}^{\prime}\right)^{2}\left[1+\left(2 \frac{\kappa_{5}^{\prime}}{\kappa_{4}^{\prime}}-\frac{\kappa_{4}}{\kappa_{3}}+C_{2}\right) r\right]+g_{z 2}(z) r^{2}+\ldots
\end{aligned}
$$

For larger values of $p$ we would obtain more exotic variants of regular horizons.

\section{EXAMPLE: KERR-NEWMAN METRIC}

To illustrate the above properties, let us consider the simplest and, at the same time, one of the most physically important cases - the Kerr-Newman metric. In the Boyer-Lindquist coordinates this metric can be written as [25]

$$
\begin{aligned}
d s^{2} & =-\left(1-\frac{2 M r-Q^{2}}{\Sigma}\right) d t^{2}-\frac{2 a\left(2 M r-Q^{2}\right) \sin ^{2} \Theta}{\Sigma} d t d \phi+\frac{\Sigma}{\Delta} d r^{2}+\Sigma d \Theta^{2}+ \\
& +\left(r^{2}+a^{2}+\frac{\left(2 M r-Q^{2}\right) a^{2} \sin ^{2} \Theta}{\Sigma}\right) \sin ^{2} \Theta d \phi^{2}
\end{aligned}
$$

where $a$ is the angular momentum parameter, $Q$ is the electric charge, $\Sigma \equiv r^{2}+a^{2} \cos ^{2} \Theta$,

$$
\Delta=r^{2}-2 M r+a^{2}+Q^{2}=\left(r-r_{+}\right)\left(r-r_{-}\right)
$$

where $r_{ \pm}=M \pm \sqrt{M^{2}-Q^{2}}$ are the roots of equation $\Delta=0$. The larger root $r_{+}$corresponds to the event horizon. 
The expression for the metric can be rewritten in the form (6) with

$$
\begin{aligned}
\omega & =\frac{a\left(2 M r-Q^{2}\right)}{\Sigma\left(r^{2}+a^{2}\right)+\left(2 M r-Q^{2}\right) a^{2} \sin ^{2} \Theta}=\frac{a\left(2 M r-Q^{2}\right)}{\left(r^{2}+a^{2}\right)^{2}-a^{2} \Delta \sin ^{2} \Theta}, \\
N^{2} & =\frac{\Delta \Sigma}{\left(r^{2}+a^{2}\right)^{2}-a^{2} \Delta \sin ^{2} \Theta} .
\end{aligned}
$$

Two cases should be considered separately.

A. Non-extremal horizon, $M^{2}>a^{2}+Q^{2}$

Let us consider the near-horizon region, $r \rightarrow r_{+}$. Then, the proper distance between the points with coordinates $r$ and $r_{+}$is

$$
n \approx 2 \sqrt{\Sigma_{+}} \frac{\sqrt{r-r_{+}}}{\sqrt{r_{+}-r_{-}}}
$$

where $\Sigma_{+} \equiv r_{+}^{2}+a^{2} \cos ^{2} \Theta$. Correspondingly, eq. (105) gives us

$$
N \approx \kappa n
$$

where

$$
\kappa=\frac{r_{+}-r_{-}}{2\left(r_{+}^{2}+a^{2}\right)}
$$

is the surface gravity of the Kerr-Newman black hole. It follows from (104) that

$$
\omega-\omega_{H} \approx a\left(r-r_{+}\right)\left[\frac{2\left(M-2 r_{+}\right)}{\left(r_{+}^{2}+a^{2}\right)^{2}}+\frac{a^{2} \sin ^{2} \Theta\left(r_{+}-r_{-}\right)}{\left(r_{+}^{2}+a^{2}\right)^{3}}\right]
$$

where $\omega_{H}=\omega\left(r=r_{+}\right)=a\left(r_{+}^{2}+a^{2}\right)^{-1}=$ const. Thus the first correction to $\omega_{H}$ has the order $r-r_{+} \sim N^{2}$, in agreement with $(24)$.

\section{B. Extremal horizon, $M^{2}=a^{2}+Q^{2}$}

Then, $r_{+}=r_{-}, \Delta=\left(r-r_{+}\right)^{2}$. The corrections to $\omega_{H}$ have the order $r-r_{+}$again. However, now $r-r_{+} \sim N$. Moreover, the term with angular dependence in (109) vanishes, so $\omega-\omega_{H} \approx \tilde{\omega}_{1} N$ in agreement with (31), where $\tilde{\omega}_{1}, \omega_{1}=$ const according to (73).

To obtain the triple horizon, one may introduce into consideration the cosmological term. However, we will not list the corresponding rather cumbersome expressions since the the triple root does not correspond in this case to the black hole horizon, as the region with $r>r_{+}$is a 
cosmological one, with the positive factor in $d s^{2}$ at $d t^{2}$.

\section{ON-HORIZON STRUCTURE OF THE EINSTEIN TENSOR}

The important property of the horizon consists in that the Einstein tensor in the OZAMO frame becomes diagonal in the horizon limit. If the Einstein equations are satisfied, this leads to important constraints on the possible form of the equation of state near the horizon. Diagonality of the Einstein tensor was demonstrated in [4] for non-extremal horizons by direct calculations in the tetrad that does not coincide with that of OZAMO and is not orthogonal, although the discrepancy becomes negligible in the limit under consideration. Meanwhile, the lack of orthogonality generates its own corrections which are hard to control.

Here, we show that the Einstein tensor indeed becomes diagonal in the orthogonal frame attached to an OZAMO. To this end, we use two different approaches. First, we calculate the components of this tensor directly like it was done in [4], but for a different, exactly orthonormal, tetrad. Second, we show that the structure of the Einstein tensor can be understood if one takes into account the relationship between OZAMO and FZAMO.

\section{A. Direct calculation}

We want to establish the asymptotic structure of the Einstein tensor in the OZAMO frame, denoted by tildes. The general expressions, though lengthy, are quite manageable and are given in appendix. We are interested in the difference

$$
\begin{aligned}
\tilde{G}_{0}^{0}-\tilde{G}_{2}^{2}= & \frac{g_{\phi \phi}}{2 g_{z z}}\left(\frac{\partial_{z} \omega}{N}\right)^{2}+\frac{1}{2}\left\{D_{n}^{2} g_{z z}+D_{n}^{2} g_{\phi \phi}\right\}+ \\
& -\frac{1}{2} \frac{\partial_{n} N}{N} \partial_{n}\left(\gamma_{\phi}+\gamma_{z}\right)-\frac{1}{2 g_{z z}} \frac{\partial_{z} N}{N} \partial_{z}\left(\gamma_{\phi}-\gamma_{z}\right)-\frac{1}{g_{z z}} \frac{\partial_{z}^{2} N}{N}
\end{aligned}
$$

and the two non-zero off-diagonal components $\tilde{G}_{1}^{0}$ and $\tilde{G}_{3}^{2}$ (for explicit expressions and definition of operator $D_{a}^{2}(136)$ see appendix; the others are zero due to symmetry). It was shown in [4] that in the horizon limit for the non-extremal case all the three quantities tend to zero, so that the Einstein tensor diagonalizes. Let us show now that the regularity conditions derived above imply that it actually diagonalizes for arbitrary horizons, either extremal or not, in a quite general manner.

We start from the $\tilde{G}_{0}^{0}-\tilde{G}_{2}^{2}$ term. Rewriting the parts with derivatives by $n$ in terms of 
$\partial_{r}=\sqrt{A} \partial_{n}$, we get

$\tilde{G}_{0}^{0}-\tilde{G}_{2}^{2}=\frac{1}{2 g_{z z}}\left\{g_{\phi \phi}\left(\frac{\partial_{z} \omega}{N}\right)^{2}-\frac{\partial_{z} N}{N} \partial_{z}\left(\gamma_{\phi}-\gamma_{z}\right)-2 \frac{\partial_{z}^{2} N}{N}\right\}+\frac{A(r)}{2}\left[D_{r}^{2} g_{\phi \phi}+D_{r}^{2} g_{z z}+\partial_{r} \ln \frac{A}{N^{2}}\right]$

The second term here is $O\left(N^{2}\right)$, as $A \sim N^{2}$ and therefore $\partial_{r} \ln \left(A / N^{2}\right)=O(1)$, for arbitrary horizons.

- In the non-extremal and extremal cases the expression in the braces is also $O\left(N^{2}\right)$ due to the regularity conditions in the form (74);

- In order to see that the same holds in the ultra-extremal case, we rewrite it as

$$
\begin{aligned}
\tilde{G}_{0}^{0}-\tilde{G}_{2}^{2} & =\frac{1}{2 g_{z z}}\left\{g_{\phi \phi}\left(\frac{\partial_{z} \omega}{N}\right)^{2}+\partial_{z} \gamma_{\phi} \frac{\partial_{z} N^{2}}{N^{2}}\right\}+ \\
& +\frac{1}{4 g_{z z}}\left\{\partial_{z}\left(\gamma_{z}-3 \gamma_{\phi}\right) \frac{\partial_{z} N^{2}}{N^{2}}+\left(\frac{\partial_{z} N^{2}}{N^{2}}\right)^{2}-2 \frac{\partial_{z}^{2} N^{2}}{N^{2}}\right\}+O\left(N^{2}\right) .
\end{aligned}
$$

The expression in the first braces is $O\left(N^{2}\right)$ due to condition $2(77)$, and the one in the second braces is also $O\left(N^{2}\right)$ due to condition $4(79)$.

Thus for all horizons

$$
\tilde{G}_{0}^{0}-\tilde{G}_{2}^{2}=O\left(N^{2}\right)
$$

Likewise the off-diagonal components in terms of $r$ can be put down in the form

$$
\begin{aligned}
& \frac{1}{\sqrt{g_{\phi \phi}}} \tilde{G}_{1}^{0}=-\frac{1}{4 g_{z z}}\left\{\partial_{z}\left(\gamma_{z}-3 \gamma_{\phi}\right) \cdot \partial_{z} \omega+\frac{\partial_{z} N^{2}}{N^{2}} \partial_{z} \omega-2 \partial_{z}^{2} \omega\right\} \frac{1}{N}+O(N) ; \\
& \sqrt{g_{z z}} \tilde{G}_{2}^{3}=\left\{\frac{g_{\phi \phi}}{2} \partial_{r} \omega \cdot \partial_{z} \omega-N \partial_{r} \partial_{z} N\right\} \frac{\sqrt{A}}{N^{2}}+O(N) .
\end{aligned}
$$

In the non-extremal and extremal cases the terms in braces are $O\left(N^{2}\right)$ due to conditions (74), and in the ultra-extremal case the same result follows from conditions 1,3 and $5(76,78,80)$. Note that the finiteness of scalar invariants only, which leads to $\partial_{z} \omega=O(N)$, is not sufficient for $\tilde{G}_{1}^{0}$ to turn to zero; the converse result was mistakenly obtained in [4] due to the use of the tetrad which is not exactly orthonormal.

As the result, for all horizons

$$
\tilde{G}_{1}^{0}, \tilde{G}_{3}^{2}=O(N)
$$




\section{B. Kinematic origin of the on-horizon structure of the Einstein tensor}

Let us, following our general logic, assume that all components of the curvature tensor in the FZAMO frame $\left\{e_{\mu}\right\}$ are finite. Both groups of observers - FZAMOs and OZAMOs - are related by local Lorentz boosts (57). Now, we will show that these two circumstances entail a rather general form of the constraints on the structure of the Einstein tensor in the OZAMO frame $\left\{h_{\mu}\right\}$ (51). For simplicity, we will restrict our consideration here to FZAMOs falling relative to OZAMOs exactly in the radial direction, so that $\theta=0$ and the second step (56) in building the FZAMO tetrad is omitted. Recall that tildes denote tensors calculated in the OZAMO frame, and hats - those in FZAMO frame.

Indeed, let us write down the boost (57) in the form

$$
e_{(\xi)}=\mathrm{x}_{\xi}^{\eta} h_{(\eta)}, \quad \xi, \eta=0,2
$$

where

$$
\mathrm{x}_{\xi}^{\eta}=\gamma\left(\begin{array}{ll}
1 & v \\
v & 1
\end{array}\right)
$$

and the Lorentz factor is

$$
\gamma \equiv \frac{1}{\sqrt{1-v^{2}}}=\frac{E}{N}
$$

which follows from the conservation of energy and the fact that the conserved angular momenta of both observers are equal to zero.

Then, we can calculate $\hat{G}_{00}, \hat{G}_{22}$ in terms of the OZAMO frame and take the horizon limit, in which $N \rightarrow 0, v \rightarrow 1$. After some elementary algebra, one finds that

$$
\begin{aligned}
& \hat{G}_{00}=\tilde{G}_{\xi \eta} \mathrm{x}_{0} \mathrm{x}_{0}{ }_{0}=\gamma^{2} \tilde{G}_{00}+2 \gamma v \tilde{G}_{02}+\gamma^{2} v^{2} \tilde{G}_{22}, \\
& \hat{G}_{22}=\tilde{G}_{\xi \eta \mathrm{x}_{2} \mathrm{x}_{2}}=\gamma^{2} \tilde{G}_{22}+2 \gamma v \tilde{G}_{02}+\gamma^{2} v^{2} \tilde{G}_{00} .
\end{aligned}
$$

In the horizon limit then, taking into account that due to symmetry (see Eq. (139) in Appendix) $\tilde{G}_{02}=0$

$$
\left(\hat{G}_{2}^{2}-\hat{G}_{0}^{0}\right) \equiv\left(\hat{G}_{22}+\hat{G}_{00}\right) \approx 2 \gamma^{2}\left(\tilde{G}_{22}+\tilde{G}_{00}\right)
$$

so, the finiteness of $\left(\hat{G}_{0}^{0}-\hat{G}_{2}^{2}\right)$ requires that

$$
\left(\tilde{G}_{0}^{0}-\tilde{G}_{2}^{2}\right)=O\left(\gamma^{-2}\right)=O\left(N^{2}\right)
$$


In a similar way one can easily show that

$$
\hat{G}_{01}=\gamma \tilde{G}_{01}, \quad \hat{G}_{23}=\gamma \tilde{G}_{23}
$$

so the finiteness of $\hat{G}_{\mu \nu}$ leads also to

$$
\tilde{G}_{01}=O(N) \text { and } \quad \tilde{G}_{32}=O(N) .
$$

The results coincide with those obtained above by direct calculations in the OZAMO frame.

\section{SUMMARY AND CONCLUSION}

We investigated the restrictions imposed on the metric by the conditions of regularity of increasing strength: boundedness of the Ricci scalar, boundedness of quadratic scalar invariants, boundedness of tetrad components of the curvature tensor in a frame attached to a falling observer. The results apply to generic dirty axially symmetric rotating black holes.

Starting with the non-extremal metric written in terms of proper distance to the horizon $n$, we saw in particular, that the regularity conditions demand the metric to be expanded in terms of the quasiglobal coordinate $r \sim n^{2}$ rather than $n$ : for scalar invariants and tetrad components of the curvature tensor to be bounded it is necessary that

$$
g_{\mu \nu}=g_{\mu \nu}^{(h)}+g_{\mu \nu}^{(1)} r+g_{\mu \nu}^{(2)} r^{2}+o\left(r^{2}\right)
$$

For extremal and ultra-extremal metrics we wrote the expansions of metric functions in terms of $r$ from the very beginning. The conditions of regularity obtained in all cases, assuming the expansions in terms of integer powers of $r$, are collected in two tables: I in terms of asymptotic behavior of metric functions and in II in terms of coefficients of their expansions by $r$ (37).

\begin{tabular}{|l||c|c|c|}
\hline What is bounded & Non-extr., $p=1$ & Extr., $p=2$ & Ultra-extr., $p>2$ \\
\hline \hline Ricci scalar & $\partial_{z} \omega=O\left(N^{2}\right)$ & \multicolumn{2}{|c|}{$\partial_{z} \omega=O(N)$} \\
\hline Quadratic invariants & $\kappa=$ const & \multicolumn{2}{|c|}{$\kappa=0^{*}$} \\
\hline $\begin{array}{l}\text { Curvature tensor } \\
\text { in FZAMO frame }\end{array}$ & $\partial_{z} \ln N^{2}, \partial_{z} \omega=O\left(N^{2}\right)$ & six conditions \\
\hline
\end{tabular}

TABLE I: The restrictions on metric for different types of horizons that follow from conditions of regularity of increasing strength; each line gives the conditions for the corresponding quantity to be bounded, additional to those stated in all the lines above (*: holds by definition).

In the extremal case those imply the uniformity of the asymptotic behavior of the lapse 


\begin{tabular}{|l||c|c|c|}
\hline What is bounded & Non-extr., $p=1$ & Extr., $p=2$ & Ultra-extr., $p=3$ \\
\hline \hline Ricci scalar & $\omega_{H}^{\prime}=0$ & $\omega_{H}^{\prime}=0$ & $\omega_{H}^{\prime}=\omega_{1}^{\prime}=0$ \\
\hline Quadratic invariants & $\kappa^{\prime}=0$ & - & - \\
\hline $\begin{array}{l}\text { Curvature tensor } \\
\text { in FZAMO frame }\end{array}$ & - & $\omega_{1}^{\prime}=0$, & $\omega_{2}^{\prime}=0, \quad \kappa_{3}^{\prime}=0 ;$ \\
\hline
\end{tabular}

TABLE II: The same restrictions as in table I in terms of expansion coefficients of the metric functions in powers of the quasiglobal coordinate $r(37)$ and for $p=3$ as an example of ultra-extremal metric.

function $N$ and parameter $\omega$ near the horizon, that generalize the weakest but most obvious restrictions on the surface gravity $\kappa=$ const and angular velocity of rotation $\omega_{H}=$ const for non-extremal horizons. In the ultra-extremal case the same can be said in loose terms, however, the explicit form of conditions (76-81) is more complicated and cannot be unambiguously interpreted in such a way.

If we write out explicitly the expansions for the lapse function and the coefficient $\omega$ in terms of $r$ for different kinds of horizons, the results read simply as follows:

$$
\begin{aligned}
p=1: \quad N^{2} & =\underline{\kappa^{2}} r+\kappa_{2}(z) r^{2}+o\left(r^{2}\right) ; \\
\omega & =\underline{\omega_{H}}+\omega_{1}(z) r+o(r) ; \\
p=2: \quad N^{2} & =\underline{\kappa_{2}} r^{2}+\underline{\kappa_{3}} r^{3}+\kappa_{4}(z) r^{4}+O\left(r^{5}\right) ; \\
\omega & =\underline{\omega_{H}}+\underline{\omega_{1}} r+\omega_{2}(z) r+O\left(r^{2}\right) ; \\
p=3: \quad N^{2} & =\underline{\kappa_{3}} r^{3}+\underline{\kappa_{4}} r^{4}+\underline{\kappa_{5}} r^{5}+\kappa_{6}(z) r^{6}+o\left(r^{7}\right) ; \\
\omega & =\underline{\omega_{H}}+\underline{\omega_{1}} r+\underline{\omega_{2}} r^{2}+\omega_{3}(z) r^{3}+o\left(r^{3}\right),
\end{aligned}
$$

however for ultraextremal case $(p \geq 3)$ exotic variants are also possible, given in Sec. IV B 5 . The coefficients that are constants are underlined.

It is instructive to compare the relationships between aforementioned properties, analyticity and possibility to cross the horizon in the spherically symmetric space-times and in the present case. For a spherically symmetric black hole the analyticity of the metric near the horizon guarantees that an observer falling into the black hole does not experience infinite tidal forces [15]. In the case of rotating black holes the situation is more subtle. The metric depends on two spatial variables. In the vicinity of the horizon, this dependence drops out from the asymptotic expressions in the main approximation and, moreover, within this approximation the metric can be analytical with respect to the quasiglobal coordinate. Nonetheless, the presence of the dependence on $z$ in the next corrections can give rise to infinite components of the curvature tensors for FZAMO for (ultra)extremal horizons. Say, let in (128) the coefficient $\kappa_{2}$ be still constant but $\kappa_{3}=\kappa_{3}(z)$. Then, in the main approximation the lapse function looks analytical 
in term of $r$ but infinite curvature components arise due to the term with $\kappa_{3}$. Therefore, it turned out that such fundamental conditions like rigid rotation and analiticity of the metric in the immediate vicinity of the horizon are necessary but, in contrast to non-extremal ones, not sufficient for the metric near the (ultra)extremal horizon to be completely regular.

It was shown in [4] that in the vicinity of a non-extremal horizon the Einstein tensor in the (orbital) ZAMO frame diagonalizes. We generalized this result to horizons of all degrees of extremality and showed that if the tetrad components of the curvature tensor in the frame attached to a falling observer stay finite, then

$$
\tilde{G}_{0}^{0}-\tilde{G}_{2}^{2}=O\left(N^{2}\right), \quad \tilde{G}_{1}^{0}, \tilde{G}_{3}^{2}=O(N)
$$

\section{Acknowledgments}

The work of O. Z. was supported in part by the Cosmomicrophysics section of the Programme of the Space Research of the National Academy of Sciences of Ukraine.

\section{APPENDIX: EINSTEIN TENSOR IN ZAMO FRAME}

The explicit expressions for the Einstein tensor in the OZAMO frame (51), denoted by tildes, in terms of the proper distance to the horizon $n$ are as follows. The diagonal ones are

$$
\begin{aligned}
\tilde{G}_{0}^{0} & =\frac{g_{\phi \phi}}{4}\left[\left(\frac{\partial_{n} \omega}{N}\right)^{2}+\frac{1}{g_{z z}}\left(\frac{\partial_{z} \omega}{N}\right)^{2}\right]+\frac{1}{2}\left\{D_{n}^{2} g_{z z}+D_{n}^{2} g_{\phi \phi}+\frac{1}{g_{z z}} D_{z}^{2} g_{\phi \phi}\right\}+ \\
& +\frac{1}{4}\left[\partial_{n} \gamma_{\phi} \partial_{n} \gamma_{z}-\frac{1}{g_{z z}} \partial_{z} \gamma_{\phi} \partial_{z} \gamma_{z}\right] \\
\tilde{G}_{2}^{2} & =\frac{g_{\phi \phi}}{4}\left[\left(\frac{\partial_{n} \omega}{N}\right)^{2}-\frac{1}{g_{z z}}\left(\frac{\partial_{z z} \omega}{N}\right)^{2}\right]+\frac{1}{2}\left\{\frac{1}{g_{z z}} D_{z}^{2} g_{\phi \phi}\right\}+ \\
& +\frac{1}{4}\left[\partial_{n} \gamma_{\phi} \partial_{n} \gamma_{z}-\frac{1}{g_{z}} \partial_{z} \gamma_{\phi} \partial_{z} \gamma_{z}\right]+\frac{1}{2} \frac{\partial_{n} N}{N} \partial_{n}\left(\gamma_{\phi}+\gamma_{z}\right)+\frac{1}{2 g_{z z}} \frac{\partial_{z} N}{N} \partial_{z}\left(\gamma_{\phi}-\gamma_{z}\right)+\left[\frac{1}{g_{z z}} \frac{\partial_{z}^{2} N}{N}\right] \\
\tilde{G}_{1}^{1} & =-\frac{3 g_{\phi \phi}}{4}\left[\left(\frac{\partial_{n} \omega}{N}\right)^{2}+\frac{1}{g_{z z}}\left(\frac{\partial_{z} \omega}{N}\right)^{2}\right]+\frac{1}{2}\left\{D_{n}^{2} g_{z z}\right\}+ \\
\tilde{G}_{3}^{3} & =-\frac{g_{\phi \phi}}{4}\left[\left(\frac{\partial_{n} \omega}{N}\right)^{2}-\frac{1}{g_{z z}}\left(\frac{\partial_{z} \omega}{N}\right)^{2}\right]+\frac{1}{2}\left\{D_{n} \gamma_{z}-\frac{1}{2 g_{z z}} \frac{\partial_{z} N}{N} \partial_{z} \gamma_{z}+\left[\frac{\partial_{n}^{2} N}{N}+\frac{1}{g_{z z}} \frac{\partial_{z}^{2} N}{N}\right]\right. \\
& +\frac{1}{2} \frac{\partial_{n} N}{N} \partial_{n} \gamma_{\phi}+\frac{1}{2 g_{z z}} \frac{\partial_{z} N}{N} \partial_{z} \gamma_{\phi}+\left[\frac{\partial_{n}^{2} N}{N}\right]
\end{aligned}
$$


where the notation is used

$$
D_{a}^{2} g_{\xi}=\frac{1}{\sqrt{g_{\xi}}} \partial_{a} \frac{\partial_{a} g_{\xi}}{\sqrt{g_{\xi}}}, \quad a=n, z, \quad \xi=\phi \phi, z z
$$

the off-diagonal components

$$
\begin{aligned}
& \frac{1}{\sqrt{g_{\phi \phi}}} \tilde{G}_{1}^{0}= \frac{1}{2}\left[\frac{\partial_{n}^{2} \omega}{N}+\right. \\
&\left.+\frac{1}{g_{z}} \frac{\partial_{z}^{2} \omega}{N}\right]-\frac{1}{2}\left[\frac{\partial_{n} N}{N} \frac{\partial_{n} \omega}{N}+\frac{1}{g_{z}} \frac{\partial_{z} N}{N} \frac{\partial_{z} \omega}{N}\right] \\
&+\frac{1}{4}\left[\frac{\partial_{n} \omega}{N} \partial_{n}\left(3 \gamma_{\phi}+\gamma_{z}\right)+\frac{1}{g_{z z}} \frac{\partial_{z} \omega}{N} \partial_{z}\left(3 \gamma_{\phi}-\gamma_{z}\right)\right] \\
& \sqrt{g_{z z}} \tilde{G}_{3}^{2}=\frac{g_{\phi \phi}}{2} \frac{\partial_{n} \omega}{N} \frac{\partial_{z} \omega}{N}-\frac{\partial_{n} \partial_{z} N}{N}-\frac{1}{2} \frac{\partial_{n} \partial_{z} g_{\phi \phi}}{g_{\phi \phi}}+\frac{1}{2} \frac{\partial_{z} N}{N} \partial_{n} \gamma_{z}+\frac{1}{4 g_{z z}} \partial_{z} \gamma_{\phi} \partial_{n}\left(\gamma_{\phi}+\gamma_{z}\right) ; \\
& \tilde{G}_{2}^{0}=\tilde{G}_{3}^{0}=\tilde{G}_{2}^{1}=\tilde{G}_{3}^{1}=0 .
\end{aligned}
$$

[1] J. M. Bardeen, B. Carter and S. W. Hawking, Commun. Math. Phys. 31, 161 (1973).

[2] S. W. Hawking, Commun. Math. Phys. 33, 323 (1973).

[3] A. J. M. Medved, D. Martin and M. Visser, Class. Quant. Grav. 21, 3111 (2004).

[4] A. J. M. Medved, D. Martin and M. Visser, Phys. Rev. D. 70, 024009 (2004).

[5] S. W. Hawking, G. T. Horowitz, S. F. Ross, Phys. Rev. D 51, 4302 (1995); C. Teitelboim, ibid. 51, 4315 (1995); G. W. Gibbons, R. E. Kallosh, ibid. 51, 2839 (1995).

[6] F. Pretorius, D. Vollick, W. Israel, Phys. Rev. D 57, 6311 (1998).

[7] J. P. S. Lemos and O. B. Zaslavskii, Phys. Lett. B 695, 37 (2011).

[8] M. Banados, J. Silk, S. M. West, Phys. Rev. Lett. 103, 111102 (2009).

[9] O. B. Zaslavskii, Phys. Rev. D 82, 083004 (2010).

[10] R. Meinel, Class. Quant. Grav. 23, 1359 (2006).

[11] J. P. S. Lemos and O. B. Zaslavskii, Phys. Rev. D 79, 044020 (2009).

[12] Black Holes: The Membrane Paradigm, edited by K. S. Thorne, R. H. Price, and D. A. Macdonald (Yale University Press, London, 1986).

[13] V. Pravda and O. B. Zaslavskii, Class. Quant. Grav. 22, 5053 (2005).

[14] O. B. Zaslavskii, Phys. Rev. D 76, 024015 (2007).

[15] K. A. Bronnikov, E. Elizalde, S. D. Odintsov, O. B. Zaslavskii, Phys. Rev. D 78, 064049 (2008).

[16] G. T. Horowitz and S. F. Ross, Phys. Rev. D 56, 2180 (1997).

[17] G. T. Horowitz and S. F. Ross, Phys. Rev. D 57, 1098 (1998).

[18] S. W. Hawking and G. F. Ellis, The Large Scale Structure of Space-time(Cambridge University 
Press, Cambridge, England, 1973).

[19] G. F. R. Ellis and B. G. Schmidt, Gen. Relativ. Gravit. 8, 915 (1977).

[20] E. Poisson, A Relativist's Toolkit (Cambridge University Press, Cambridge, 2004).

[21] K. A. Bronnikov, Phys. Rev. D 64, 064013 (2001).

[22] K. A. Bronnikov, G. Clément, C. P. Constantinidis, and J. C. Fabris, Gravitation Cosmol. 4, 128 (1998); Phys. Lett. A 243, 121 (1998).

[23] O. B. Zaslavskii, Phys. Rev. D 85, 024029 (2012).

[24] J. Bardeen, W. H. Press, and S. A. Teukolsky, Astrophys. J. 178, 347 (1972).

[25] G. W. Gibbons and S. W. Hawking, Phys. Rev. D, 15, 2738 (1977). 